\title{
Reliability analysis and prediction of the failure process of some traction converters
}

\author{
M. Guida ${ }^{1,2}$, P. Poli ${ }^{3} \&$ G. Pulcini ${ }^{2}$ \\ ${ }^{I}$ Department of Information Engineering, Electrical Engineering and \\ Applied Mathematics, University of Salerno, Italy \\ ${ }^{2}$ Istituto Motori, National Research Council, Italy \\ ${ }^{3}$ AnsaldoBreda SpA, Italy
}

\begin{abstract}
This paper studies the failure process of the traction converters mounted on some trains produced by AnsaldoBreda SpA and operating in the district of the city of Naples. Each train is equipped with three traction converters and 23 new trains were monitored from their entry in service over a time period of about four years. A preliminary analysis of the observed failure data, based on a nonparametric estimation procedure, showed an overall decreasing trend of the failure intensity during the monitoring period, as it is often observed during the early life of complex systems undergoing minimal repair. As such, the failure process of converters is assumed to be a non-homogeneous Poisson process (NHPP) over the covered mileage. Then, NHPP with different decreasing intensity functions are considered, and the process that better fits the observed data, namely the log-linear process, is chosen to analyse the observed failure process. Maximum likelihood estimate of the process parameters is then obtained, from which several reliability characteristics are estimated under the conservative hypothesis that the failure intensity remains constant beyond the end of the observation period. Finally, the probability distribution of the annual mileage of the train is derived, that allows the reliability characteristics to be estimated in terms of the calendar time, too.

Keywords: traction converters, reliability analysis, nonhomogeneous Poisson process, annual mileage distribution.
\end{abstract}




\section{Introduction}

The frequency converter is one of the main parts of the traction power supply system (TPSS) and converts adequate traction power from the power company to the electric vehicle. Due to the impact of its failures on the reliability of the whole TPSS, more converters are mounted on each vehicle in order to guarantee a good redundancy in case of failure and give the opportunity, using dedicated control logics, to interlace them in order to reduce the harmonic content of the absorbed current.

In this paper, the failure of the traction converters mounted on some trains produced by AnsaldoBreda SpA and operating in the district of the city of Naples is analyzed. Each train is equipped with three traction converters which, as shown in Figure 1, are fed by the high-voltage grid power line through one pantograph and each converter supplies two three-phase asynchronous traction motors, namely "M1" and "M2".

The traction converters are very complex systems, constituted by several subsystems among which: the Hall effect current transducer, the voltage transducer (that measures the input voltage to the converter), the voltage transducer (that measures the input voltage to the inverter modules), the pre-charge contactor, a number of resistors and six different types of electronics boards, the traction control unit (TCU) (that is fed by the low voltage system and manage the tractive effort, acceleration and braking), three IGBT (insulated gate bipolar transistor) inverter modules (that power the traction motors), and the brake chopper (that allows the energy returned by the traction motors to be dissipated on the braking resistor).

A fleet of new trains was monitored during a time period of about 4 years, and a failure mode and effects analysis was carried out by AnsaldoBreda in order to detect the most relevant problems and to track converter reliability during the operating period. On the basis of the data set, the objective of the present paper is to specifically address the modeling of the converter failure process, and to derive reliability measures useful to the manufacturer, whilst the analysis of

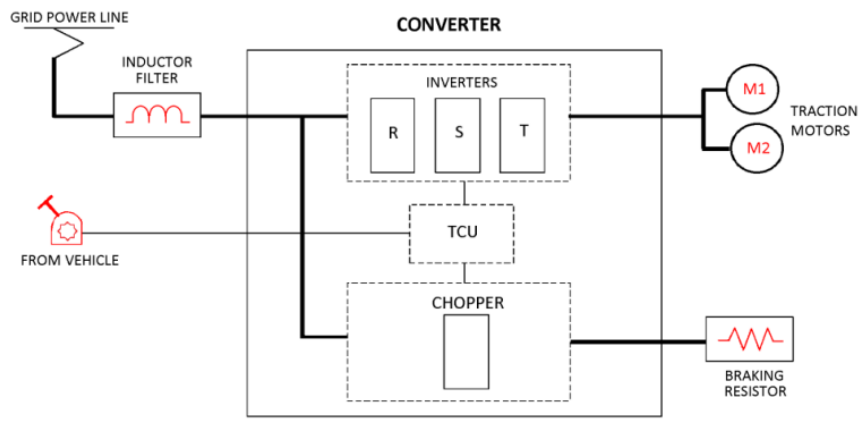

Figure 1: Block diagram of the traction drive. 
maintenance times and possible maintenance actions to perform on this system is beyond the scope of the paper. In this respect, it is to be noted that the converter is a complex system whose faults are usually due to the failure of a single (elementary) part, and whose intended function is generally restored by just replacing the single failed part by a new one. As such, it is reasonable to assume that, upon failure, the converter undergoes a "minimal repair", which (instantaneously) brings it back to an as-good-as-before condition. This assumption naturally leads to the class of non-homogeneous Poisson processes (NHPP). In particular, in the present application, the process domain is better represented by "covered mileage" than by "operating time", as mileage is the variable that better measures the converter usage.

A preliminary analysis, based on a non-parametric estimation procedure, seems to show an overall decreasing trend of the failure intensity during the observation period. This behavior appears to be in keeping with what is often observed in the initial phase of the operative life of a system, where the substitution of the early-failing (week) components with new ones which are likely to be free from manufacturing faults, makes the failure intensity to decrease. Note that the overall decreasing of the intensity function is also partly due to some external adjustments made to the converters during the initial development phase of the whole train, which result in a reduction of the stress acting on the converters.

Then, a number of NHPP with different decreasing intensity function are considered, such as the power-law process [1], the log-linear process [2] and the Musa-Okumoto process [3], and the process that better fits the observed data, namely the log-linear process, is chosen to analyze the observed process.

Maximum likelihood estimate of the process parameters is then obtained. Several reliability characteristics, such as the expected number of failures and the probability of no failure during a future operating time, are also estimated under the conservative hypothesis that the failure intensity remains constant beyond the end of the observation period, say $X$, where the failure intensity is equal to the value it had at $X$. Finally, the probability distribution of the annual mileage of the train is derived, that allows one to estimate the reliability characteristics in terms of the calendar time too, in order to better plan the maintenance actions and to better manage the spare parts inventory.

\section{Reliability analysis of failure process}

The failure processes of 23 new trains were observed from the entry in service of each train (starting from 2009, April 1) until 2013 August 31. A total of 59 converter failures occurred during the observation period, which covers only the very early life of the systems that are designed to operate for 30-40 years, covering a distance of more than 2 million kilometers.

Table 1 gives both the total mileage (in $\mathrm{km}$ ) $X_{j}$ and the annual mileage covered by each train $j(j=1, \ldots, 23)$ until 2013 August 31. In Table 2 the mileage to converters failure $x_{i, j}\left(i=1, \ldots, n_{j}\right)$ measured from the entry in 
Table 1: Total mileage $X_{j}$ and annual mileage [in $\mathrm{km}$ ] of each train $j$.

\begin{tabular}{|l|l|c|c|c|c|c|c|}
\hline$j$ & Train \# & Total $X_{j}$ & 2009 & 2010 & 2011 & 2012 & 2013 \\
\hline 1 & 201 & 125,010 & 2538 & 0 & 70,000 & 0 & 45,132 \\
\hline 2 & 202 & 217,384 & 18,317 & 50,610 & 34,012 & 70,880 & 43,565 \\
\hline 3 & 203 & 178,234 & 14,371 & 54,534 & 36,284 & 37,402 & 35,643 \\
\hline 4 & 204 & 171,026 & 16,931 & 44,992 & 48,458 & 24,514 & 36,131 \\
\hline 5 & 206 & 109,780 & 23,507 & 30,207 & 43,093 & 12,973 & 0 \\
\hline 6 & 207 & 27,709 & 20,305 & 7,404 & 0 & 0 & 0 \\
\hline 7 & 208 & 174,214 & 21,121 & 49,439 & 25,215 & 62,377 & 16,062 \\
\hline 8 & 209 & 134,465 & 21,107 & 53,810 & 36,747 & 22,801 & 0 \\
\hline 9 & 210 & 58,158 & 20,501 & 37,657 & 0 & 0 & 0 \\
\hline 10 & 211 & 150,844 & 22,441 & 49,665 & 50,333 & 28,405 & 0 \\
\hline 11 & 212 & 135,943 & 15,314 & 49,636 & 54,319 & 16,674 & 0 \\
\hline 12 & 213 & 206,553 & 24,345 & 40,215 & 62,210 & 31,698 & 48,085 \\
\hline 13 & 214 & 188,465 & 17,659 & 60,621 & 49,620 & 45,309 & 15,256 \\
\hline 14 & 215 & 179,107 & 13,834 & 64,117 & 49,948 & 51,208 & 0 \\
\hline 15 & 216 & 184,152 & 19,603 & 54,473 & 47,117 & 51,816 & 11,143 \\
\hline 16 & 217 & 193,107 & 13,224 & 55,464 & 52,830 & 63,623 & 7966 \\
\hline 17 & 218 & 201,326 & 10,579 & 57,802 & 56,189 & 76,756 & 0 \\
\hline 18 & 219 & 221,308 & 6235 & 57,801 & 67,426 & 50,635 & 39,211 \\
\hline 19 & 220 & 220,125 & 0 & 53,800 & 60,059 & 73,027 & 33,239 \\
\hline 20 & 221 & 123,989 & 0 & 54,788 & 59,370 & 9831 & 0 \\
\hline 21 & 222 & 175,119 & 0 & 32,238 & 57,800 & 52,040 & 33,041 \\
\hline 22 & 223 & 176,867 & 0 & 25,973 & 63,454 & 72,084 & 15,356 \\
\hline 23 & 224 & 155,893 & 0 & 0 & 47,660 & 44,098 & 64,135 \\
\hline The mileages in italics refer to the case in which the train was not operating during the entire year \\
\hline
\end{tabular}

service of each train $j$ are given. Unfortunately, in the available data set, no information is given about which of the three converters mounted on the train $j$ failed at $x_{i, j}$. Thus, for example, we do not know if the two failures of train \#202 refer to the same converter or not. As such, the analysis concerning the converter unit has to be derived, as illustrated in the next section, by the analysis based on the only observable failure process, i.e. the failure process of train $j$, which is the superposition of three identical, but unidentifiable, failure processes.

\subsection{Model selection and parameter estimation}

On the basis of the above observations, we made the reasonable assumption that the three failure processes of the converters mounted on the same train are independent of each other. We also assume that, due to the complex structure of the converter and the type of repair the converter is subjected to (that generally consists of the replacement of the failed part with a new one), the repairs are 
Table 2: $\quad$ Mileage to converters failure $x_{i, j}[\mathrm{in} \mathrm{km}]$ for each train $j$.

\begin{tabular}{|c|c|c|c|c|c|c|c|c|c|}
\hline$j$ & Train \# & $x_{1, j}$ & $x_{2, j}$ & $x_{3, j}$ & $x_{4, j}$ & $x_{5, j}$ & $x_{6, j}$ & $x_{7, j}$ & $x_{8, j}$ \\
\hline 1 & 201 & 125,010 & & & & & & & \\
\hline 2 & 202 & 75,755 & 121,186 & & & & & & \\
\hline 3 & 203 & 31,000 & 168,852 & & & & & & \\
\hline 4 & 204 & 29,144 & 41,188 & & & & & & \\
\hline 5 & 206 & 34,899 & 86,385 & & & & & & \\
\hline 6 & 207 & 300 & 8376 & 19,947 & & & & & \\
\hline 7 & 208 & 61,086 & 128,915 & 129,266 & 130,246 & 130,935 & & & \\
\hline 8 & 209 & 70,681 & 93,743 & 107,997 & & & & & \\
\hline 9 & 210 & & & & & & & & \\
\hline 10 & 211 & 100,383 & & & & & & & \\
\hline 11 & 212 & 75,090 & 75,239 & & & & & & \\
\hline 12 & 213 & 25,912 & 68,599 & 182,990 & & & & & \\
\hline 13 & 214 & 12,193 & 18,798 & 18,798 & 20,350 & 58,857 & 102,550 & 108,592 & 155,126 \\
\hline 14 & 215 & & & & & & & & \\
\hline 15 & 216 & 26,632 & 31,199 & 48,875 & & & & & \\
\hline 16 & 217 & 29,687 & 39,979 & 40,032 & 67,409 & 94,064 & 116,379 & & \\
\hline 17 & 218 & 7686 & 168,959 & & & & & & \\
\hline 18 & 219 & 94,127 & 94,141 & & & & & & \\
\hline 19 & 220 & 151,494 & 210,968 & & & & & & \\
\hline 20 & 221 & 29,685 & 32,595 & 32,737 & & & & & \\
\hline 21 & 222 & 22,553 & 67,594 & & & & & & \\
\hline 22 & 223 & 6150 & 92,151 & 145,724 & & & & & \\
\hline 23 & 224 & 77,071 & 78,397 & & & & & & \\
\hline
\end{tabular}

minimal, so that the failure process of each converter is modeled by a nonhomogeneous Poisson process (NHPP) with intensity function $\lambda(x)$. As a result of these two assumptions, the observed processes are NHPP with intensity function equal to $3 \lambda(x)$.

A nonparametric estimate of the average failure intensity on mileage intervals of length $\Delta=20,000 \mathrm{~km}$ is made by counting the number of failures $N_{k}$ occurred in the $k^{\text {th }}$ interval $((k-1) \Delta, k \Delta)$ and applying the formula:

$$
\tilde{\lambda}_{k}=N_{k} /\left(3 \cdot T M_{k}\right), k=1,2, \ldots, 11,
$$

where $T M_{k}$ is the total mileage covered by all of the observed trains during the interval $((k-1) \Delta, k \Delta)$. As shown in Figure 2, the estimated intensity $\tilde{\lambda}_{k}$ is decreasing overall, thus suggesting describing the converter failure process with an NHPP with decreasing failure intensity. 


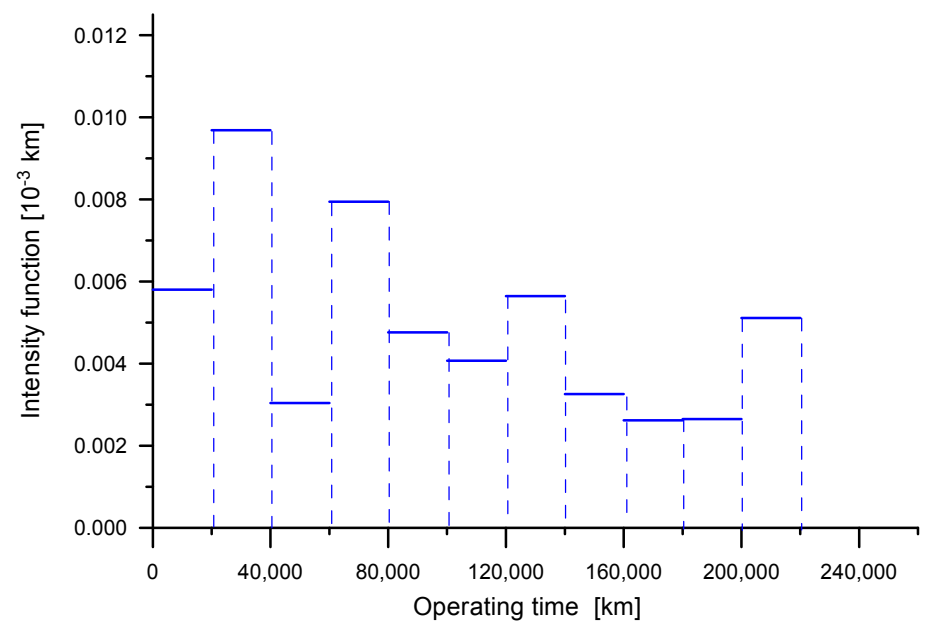

Figure 2: Nonparametric estimate of the intensity function.

The overall decreasing behavior of the intensity function is confirmed by the Laplace test that is based, for multiple time-truncated systems, on the test statistic [4]:

$$
\mathrm{LA}=\frac{\sum_{j=1}^{23} \sum_{i=1}^{n_{j}} x_{i, j}-\sum_{j=1}^{23} n_{j} X_{j} / 2}{\sqrt{\sum_{j=1}^{23} n_{j} X_{j}^{2} / 12}},
$$

which is distributed as a standard Normal variate under the null hypothesis that the intensity function is constant (no trend in the failure data). Large, negative values of LA indicate a decreasing trend. The value of the LA statistic relative to the observed data is -1.422 , that allows the null hypothesis of no trend to be rejected at the significance level of 0.10 , and also provides evidence of a decreasing trend.

Thus, three different NHPP, whose failure intensity can be decreasing, are here considered:

1. The power law process (PLP) [1], whose intensity function $\lambda(x)=$ $(\beta / \alpha)(x / a)^{\beta-1}, \alpha, \beta>0$, is decreasing for $\beta<1$. The corresponding mean number of failures is $M(x)=(x / \alpha)^{\beta}$.

2. The log-linear process (LLP) [2], whose intensity function $\lambda(x)=$ $\alpha \exp (\beta x), \alpha>0,-\infty<\beta<\infty$, is decreasing for $\beta<0$. Its mean number of failures is $M(x)=(\alpha / \beta)[\exp (\beta x)-1]$.

3. The Musa-Okumoto process (MOP) [3], with decreasing intensity function $\lambda(x)=\alpha /(\beta x+1), \alpha, \beta>0$, and $M(x)=(\alpha / \beta) \ln (\beta x+1)$.

Note that, both in the LLP and the MOP, $\alpha$ is the value of the intensity function at $x=0$. The parameters of the selected models are then estimated by maximizing the log-likelihood function relative to the observed data: 


$$
\ell(\text { data } \mid \alpha, \beta)=\sum_{j=1}^{23} \sum_{i=1}^{n_{j}} \ln \left[3 \lambda\left(x_{i, j}\right)\right]-\sum_{j=1}^{23} 3 M\left(X_{j}\right),
$$

where the multiplicative factor of 3 is due to the fact that the observed process is the superposition of three independent and identical NHPP with intensity function $\lambda(x)$. The maximum likelihood estimates (MLE) of the parameters of the selected models, the corresponding estimated log-likelihood $\hat{\ell} \equiv$ $\ell($ data $\mid \hat{\alpha}, \hat{\beta})$, and the value of the Akaike information criterion (AIC) [5] are given by:

\begin{tabular}{lcccc}
\hline NHPP & $\hat{\alpha}$ & $\hat{\beta}$ & $\hat{\ell}$ & AIC \\
\hline PLP & $192,001 \mathrm{~km}$ & 0.8574 & -710.086 & 714.086 \\
LLP & $0.760 \cdot 10^{-5} / \mathrm{km}$ & $-0.445 \cdot 10^{-5} / \mathrm{km}$ & -709.233 & 713.233 \\
MOP & $0.774 \cdot 10^{-5} / \mathrm{km}$ & $0.606 \cdot 10^{-5} / \mathrm{km}$ & -709.345 & 713.345 \\
\hline
\end{tabular}

Since, given a set of candidate models for the data, the preferred model is the one with the minimum AIC value, then the LLP is chosen and adopted for all the following analyses. Note that the difference in the AIC value with respect to the MOP is almost negligible. In Figure 3, the nonparametric estimate of the mean number of failures at the observed failure times [6] is compared to the MLE of $M(t)$ within the three selected models. For comparative purpose, also the MLE of the mean number of failures within the homogeneous Poisson process (HPP) with constant intensity function $\hat{\lambda}=0.5303 \cdot 10^{-5} / \mathrm{km}$ is depicted.

We see that both the LLP and the MOP fit the "observed" data very well, whereas the PLP is not able to satisfactorily reproduce convexity of the mean number of failures.

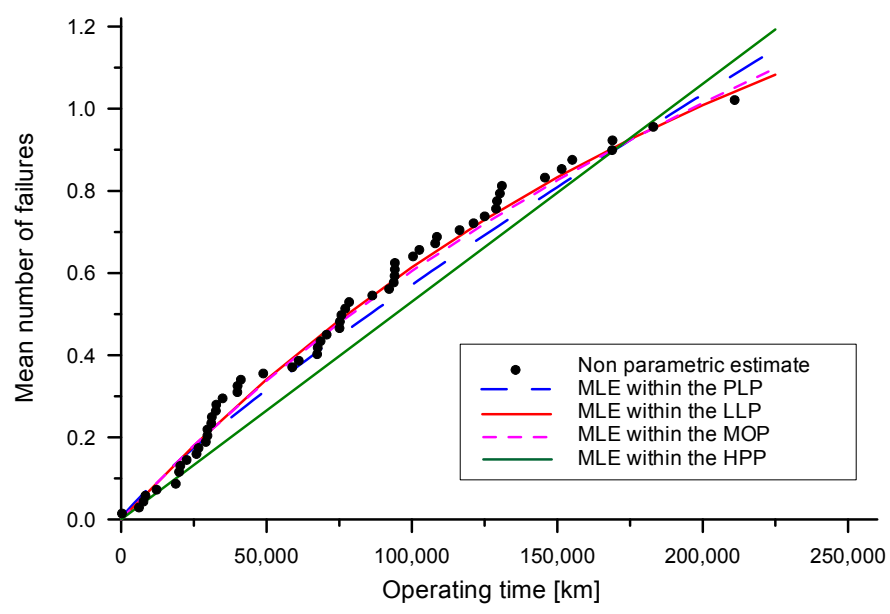

Figure 3: Nonparametric and maximum likelihood estimates of the mean number of failures. 
Since the exact distribution of the MLE of the LLP parameters is not known, confidence intervals on $\alpha$ and $\beta$ can be obtained by using asymptotic results. In particular, we first estimate the approximate covariance matrix $\mathbf{C}(\hat{\alpha}, \hat{\beta})$ which is the inverse of the estimated negative second-derivative matrix $\mathbf{J}(\hat{\alpha}, \hat{\beta})$, also known as the Fisher's information matrix (whose entries are given in the Appendix):

$$
\mathbf{C}(\hat{\alpha}, \hat{\beta})=\left(\begin{array}{cc}
0.2989 \cdot 10^{-11} & -0.3529 \cdot 10^{-11} \\
-0.3529 \cdot 10^{-11} & 0.6193 \cdot 10^{-11}
\end{array}\right) .
$$

From the matrix in eqn (4), the estimated standard deviations of $\hat{\alpha}$ and $\hat{\beta}$ are equal to $\hat{\sigma}(\hat{\alpha})=0.173 \cdot 10^{-5} / \mathrm{km}$ and $\hat{\sigma}(\hat{\beta})=0.249 \cdot 10^{-5} / \mathrm{km}$, respectively. Then, the approximate 0.90 confidence interval for the parameter $\alpha$, that is constrained to be positive, is obtained by using the lognormal approximation for $\hat{\alpha}$ :

$$
\left(\hat{\alpha} \exp \left\{ \pm z_{.05} \hat{\sigma}(\hat{\alpha}) / \hat{\alpha}\right\}\right)=\left(0.523 \cdot 10^{-5}, 1.105 \cdot 10^{-5}\right),
$$

where $z_{.05}$ is the 0.05 quantile of the standard Normal distribution. The approximate 0.90 confidence interval for the parameter $\beta$ (that is not constrained to be positive) is obtained by using the normal approximation for $\hat{\beta}$ :

$$
\left(\hat{\beta} \pm z_{.05} \hat{\sigma}(\hat{\beta})\right)=\left(-0.852 \cdot 10^{-5},-3.317 \cdot 10^{-5}\right) .
$$

From the estimated covariance matrix, the approximate standard deviation of the MLE of any function $h(\alpha, \beta)$ of the model parameters can be estimated by using the Delta method [7]:

$$
\begin{aligned}
\hat{\sigma}(\hat{h}) \cong & {\left[\left.\left(\frac{\partial h(\alpha, \beta)}{\partial \alpha}\right)^{2}\right|_{\widehat{\alpha}, \widehat{\beta}} \hat{\sigma}^{2}(\hat{\alpha})+\left.\left(\frac{\partial h(\alpha, \beta)}{\partial \beta}\right)^{2}\right|_{\widehat{\alpha}, \widehat{\beta}} \hat{\sigma}^{2}(\hat{\beta})\right.} \\
& \left.+\left.2 \frac{\partial h(\alpha, \beta)}{\partial \alpha} \cdot \frac{\partial h(\alpha, \beta)}{\partial \beta}\right|_{\widehat{\alpha}, \widehat{\beta}} \hat{\sigma}(\hat{\alpha}, \hat{\beta})\right]^{\frac{1}{2}}
\end{aligned}
$$

where $\hat{\sigma}(\hat{\alpha}, \hat{\beta})$ is the estimated covariance of $\hat{\alpha}$ and $\hat{\beta}$ (the entry $(1,2)$ of $\mathbf{C}(\hat{\alpha}, \hat{\beta}))$. Then, by using the normal or the lognormal approximation for the distribution of the MLE of $h(\alpha, \beta)$, the approximate confidence interval for $h(\alpha, \beta)$ can be easily obtained.

\section{Reliability prediction}

Once the model has been selected and its parameters have been estimated, any reliability characteristic of the converter can be estimated. In particular, we are 
firstly interested into the expected number of failures that a converter mounted on the train $j$ will experience in a future mileage interval $\left(X_{j}, X_{j}+\Delta\right)$ of width $\Delta$, and in the system reliability during the same future interval.

At this aim, we made the conservative assumption that the failure intensity $\lambda(x)$ of each converter decreases with exponential law $\lambda(x)=\alpha \exp (\beta x)$ only up to the largest observed mileage to failure, say $X_{0}=\max _{i, j}\left(x_{i, j}\right)=210,968$ $\mathrm{km}$, and from $X_{0}$ up to the (unknown) mileage at which degradation phenomena start to become relevant, it remains constant and equal to the value it has at $X_{0}$, say $\lambda_{0}=\alpha \exp (\beta \cdot 210,968)=0.2987 \cdot 10^{-5} / \mathrm{km}$.

This implies that from $X_{0}=210,968 \mathrm{~km}$ onwards, that is, during the socalled "useful life", the mean mileage between failures is constant and equal to $\operatorname{MMBF}=1 / \lambda_{0}$. Its MLE is equal to $334,780 \mathrm{~km}$.

The standard deviations of the intensity $\lambda_{0}$ are estimated by using the Delta method: $\hat{\sigma}\left(\hat{\lambda}_{0}\right)=0.110 \cdot 10^{-5} / \mathrm{km}$, being $\partial \lambda_{0} / \partial \alpha=\exp \left(\beta X_{0}\right)$ and $\partial \lambda_{0} / \partial b=$ $\alpha X_{0} \exp \left(\beta X_{0}\right)$. The approximate 0.90 confidence interval for $\lambda_{0}$, based on the lognormal approximation for the distribution of $\hat{\lambda}_{0}$, results in: $\left(0.151 \cdot 10^{-5} / \mathrm{km}\right.$, $\left.0.539 \cdot 10^{-5} / \mathrm{km}\right)$.

Likewise, the estimate of the approximate standard deviation of the MLE of the MMBF is equal to $135,520 \mathrm{~km}$, and the corresponding approximate 0.90 confidence interval is: $(185,522 \mathrm{~km}, 662,017 \mathrm{~km})$.

On the basis of the above conservative assumption on the behavior of the intensity function, the expected number of failures in the future mileage interval $\left(X_{j}, X_{j}+\Delta\right)$ is given by:

$M\left(X_{j}, X_{j}+\Delta\right)= \begin{cases}\frac{\alpha}{\beta}\left\{\exp \left[\beta\left(X_{j}+\Delta\right)\right]-\exp \left(\beta X_{j}\right)\right\}, & X_{j}+\Delta \leq X_{0} \\ \frac{\alpha}{\beta}\left\{\exp \left(\beta X_{0}\right)-\exp \left(\beta X_{j}\right)\right\}+\left(X_{j}+\Delta-X_{0}\right) \lambda_{0}, & X_{j}+\Delta>X_{0}\end{cases}$

and the system reliability relative to the same interval is $R_{j}\left(X_{j}, X_{j}+\Delta\right)=$ $\exp \left[-M\left(X_{j}, X_{j}+\Delta\right)\right]$. In addition, the probability distribution of the number of failures in $\left(X_{j}, X_{j}+\Delta\right)$ is given by:

$$
\operatorname{Pr}\left\{N_{j}\left(X_{j}, X_{j}+\Delta\right)=k\right\}=\frac{\left[M_{j}\left(X_{j}, X_{j}+\Delta\right)\right]^{k}}{k !} \exp \left[-M_{j}\left(X_{j}, X_{j}+\Delta\right)\right] .
$$

In order to better plan the maintenance actions and to better manage the spare parts inventory, it can be also useful to estimate the reliability characteristics not in terms of a future mileage interval $\Delta$, but rather in terms of a future calendar time interval $\tau$, e.g., one year, starting from the current date. To do this, it is necessary to derive the probability distribution of the mileage covered by a train during a prefixed calendar time interval.

At this aim, we use the annual mileages given in Table 1, limited to the 50 values relative to the cases in which the train was in operation from the beginning to the end of each year, to derive the probability distribution of 
the annual mileage $Z$. In particular, we assume that $Z$ is Weibull distributed with probability density function: $f(z)=(b / a)(z / a)^{b-1} \exp \left[-(z / a)^{b}\right]$, where the scale $a$ and shape $b$ parameters are positive [8]. The Weibull plot in Figure 4 shows that the Weibull distribution fits quite well the observed annual mileages (the coefficient of determination $R^{2}$ is equal to 0.981 ).

The MLE of the Weibull parameters are $\hat{a}=56,184 \mathrm{~km}$ and $\hat{b}=5.29$. By using these estimates, the Anderson-Darling (AD) goodness-of-fit test is performed to analytically check the goodness of fit of the Weibull distribution. The AD statistic $A^{2}$ results in $A^{2}=0.316$ and, when compared to the critical value of 0.737 relative to a sample of size 50 and a significance level of 0.05 [8], shows that the Weibull assumption cannot be rejected.

Thus, from the Weibull distribution on the annual mileage, the expected number of failures and the system reliability relative to a future calendar time interval of $\tau=1$ year are given by:

$$
\begin{gathered}
M_{j}\left(T_{j}, T_{j}+1\right)=\int_{0}^{\infty} M_{j}\left(X_{j}, X_{j}+z\right) \cdot f(z) \mathrm{d} z, \\
R_{j}\left(T_{j}, T_{j}+1\right)=\int_{0}^{\infty} \exp \left[-M_{j}\left(X_{j}, X_{j}+z\right)\right] \cdot f(z) \mathrm{d} z,
\end{gathered}
$$

where $T_{j}$ is the calendar date of train $j$ at the end of the truncated observed mileage $X_{j}$. Table 3 gives the MLE of the reliability characteristics of each individual converter mounted on selected trains, relative both to a future mileage of $\Delta=60,000 \mathrm{~km}$ and to a future calendar time of $\tau=1$ year. Of course, since the intensity function decreases up to $X_{0}=210,968 \mathrm{~km}$, the smaller is the current mileage $X_{j}$ of the train, the larger is the mean number of future failures and the lower is the system reliability.

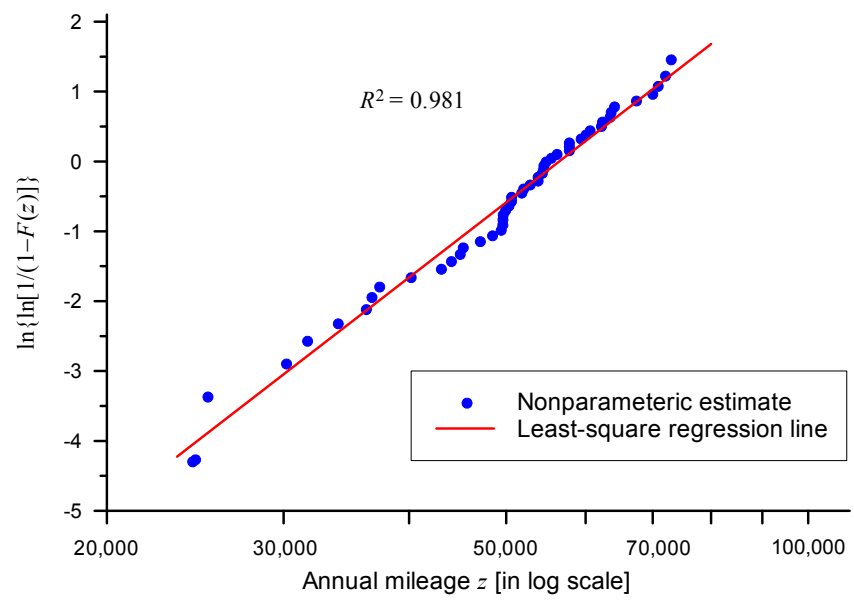

Figure 4: Weibull plot of annual mileages. 
Table 3: Reliability prediction of each individual converter.

\begin{tabular}{|l|c|c|c|c|c|}
\hline & & \multicolumn{2}{|c|}{ Mean number of failures } & \multicolumn{2}{c|}{ System reliability } \\
\cline { 3 - 6 } Train \# & $X_{j}[\mathrm{~km}]$ & $\Delta=60,000 \mathrm{~km}$ & $\tau=1$ year & $\Delta=60,000 \mathrm{~km}$ & $\tau=1$ year \\
\hline 201 & 125,010 & 0.230 & 0.201 & 0.794 & 0.818 \\
\hline 202 & 217,384 & 0.179 & 0.155 & 0.836 & 0.857 \\
\hline 207 & 27,709 & 0.354 & 0.309 & 0.702 & 0.735 \\
\hline 210 & 58,158 & 0.309 & 0.270 & 0.734 & 0.764 \\
\hline
\end{tabular}

In particular, for all trains that have currently accumulated at least 210,968 $\mathrm{km}$, so that the failure intensity of the converter is constant from here on out, the estimated mean number of failures of each individual converter during the next year is equal to $\hat{\lambda}_{0} \cdot \hat{E}\{Z\}=0,155$, being the estimated mean annual mileage $\widehat{E}\{Z\}$ equal to $51.755 \mathrm{~km} /$ year. This implies that, during the "useful life", over a fleet of 23 trains that operates regularly, the estimated mean number of converters failures during the future year is 10.67 .

Finally, the (constant) mean (calendar) time between failures MCTBF, from $X_{0}=210,968 \mathrm{~km}$ onwards, is equal to 6.47 years of uninterrupted operation. However, since each train is planned to operate for 10 hours a day and for 335 days a year, the (constant) mean (operating) time between failures MOTBF, from $X_{0}=210,968 \mathrm{~km}$ onwards, is equal to 56.665 hours or, equivalently, to 16.91 years.

\section{Conclusions}

In this paper, the failure process of the traction converters of AnsaldoBreda SpA trains operating in the district of the city of Naples has been analyzed under the hypothesis that the three converters equipping each train fail independently of each other and are minimally repaired. The observed period covers only the early life of the converters where the failure processes show a decreasing trend in the failure occurrence. The assumption that from the last observed failure onwards the failure intensity is constant is then made in order to obtain conservative prediction of the system reliability during future operating intervals, given both in terms of mileage and time.

\section{Appendix}

The log-likelihood function in eqn (4) of the LLP relative to multiple timetruncated systems is:

$$
\ell(\text { data } \mid \alpha, \beta)=N \ln 3+N \ln \alpha+\sum_{j=1}^{23} \sum_{i=1}^{n_{j}} \beta x_{i, j}-\frac{3 \alpha}{\beta} \sum_{j=1}^{23}\left[\exp \left(\beta X_{j}\right)-1\right],
$$

where $N=\sum_{j=1}^{23} n_{j}=59$ is the total number of observed failures. The elements of the estimated negative second-derivative matrix $\mathbf{J}(\hat{\alpha}, \hat{\beta})$ are: 


$$
\begin{gathered}
-\left.\frac{\partial^{2} \ell}{\partial \alpha^{2}}\right|_{\widehat{\alpha}, \widehat{\beta}}=\frac{N}{\widehat{\alpha}^{2}}, \\
-\left.\frac{\partial^{2} \ell}{\partial \beta^{2}}\right|_{\widehat{\alpha}, \widehat{\beta}}=\frac{3 \widehat{\alpha}}{\widehat{\beta}^{3}} \sum_{j=1}^{23}\left[\exp \left(\hat{\beta} X_{j}\right)\left(\hat{\beta}^{2} X_{j}^{2}-2 \hat{\beta} X_{j}+2\right)-2\right], \\
-\left.\frac{\partial^{2} \ell}{\partial \alpha \partial \beta}\right|_{\widehat{\alpha}, \widehat{\beta}}=\frac{3}{\widehat{\beta}^{2}} \sum_{j=1}^{23}\left[1-\exp \left(\hat{\beta} X_{j}\right)\left(1-\hat{\beta} X_{j}\right)\right]
\end{gathered}
$$

where the MLE of $\beta$ is the (numerical) solution of

$$
\sum_{j=1}^{23} \sum_{i=1}^{n_{j}} x_{i, j}+\frac{N}{\widehat{\beta}} \cdot \frac{\sum_{j=1}^{23}\left[\exp \left(\widehat{\beta} X_{j}\right)\left(1-\widehat{\beta} X_{j}\right)-1\right]}{\sum_{j=1}^{23}\left[\exp \left(\widehat{\beta} X_{j}\right)-1\right]}=0
$$

and $\hat{\alpha}=\frac{N \widehat{\beta}}{\left\{3 \sum_{j=1}^{23}\left[\exp \left(\widehat{\beta} X_{j}\right)-1\right]\right\}}$.

\section{References}

[1] Crow, L.H., Reliability analysis for complex, repairable systems. Reliability and Biometry, eds. F. Proschan \& R.J. Serfling, SIAM: Philadelphia, pp. 379-410, 1974.

[2] Cox, D.R. \& Lewis, P.A.W., The Statistical Analysis of Series of Events, Chapman and Hall: London, 1966.

[3] Musa, J.D. \& Okumoto, K., A logarithmic Poisson execution time model for software reliability measurement. Proc. of the $7^{\text {th }}$ International Conference on Software Engineering, IEEE Press: Piscataway, pp. 230-238, 1984.

[4] Kvaløy, J.T. \& Lindqvist, B.H., TTT-based tests for trend in repairable systems data. Reliability Engineering and System Safety, 60(1), pp. 13-28, 1998.

[5] Akaike, H., A new look at the statistical model identification. IEEE Transactions on Automatic Control, 19(6), pp. 716-723, 1974.

[6] Pulcini, G., Repairable system analysis for bounded intensity functions and various operating conditions. Journal of Quality Technology, 40(1), pp. 78-96, 2008.

[7] Oehlert, G.W., A note on the Delta method. The American Statistician, 46(1), pp. 27-29, 1992.

[8] Dodson, B. The Weibull Analysis Handbook. American Society for Quality, Quality Press: Milwaukee, 2006. 